Trauma Berufskrankh $2005 \cdot 7$ [Suppl 2]: S271-S276 DOI 10.1007/s10039-005-1037-3

Online publiziert: 4. August 2005

(c) Springer Medizin Verlag 2005

H.-J. Oestern

Klinik für Unfall- und Wiederherstellungschirurgie, Allgemeines Krankenhaus, Celle

\title{
Komplikationen bei Densfrakturen
}

- B2.1: Densspitze/Avulsionsverletzung

- B2.2: Densbasis bzw. Körper

- B2.3: Denshals

- B3: komplexe Axisverletzungen

Grauer et al. [27] schlugen eine Modifikation vor für die Unterscheidung der Typ-IIund -III-Frakturen im Hinblick auf die $\mathrm{C} 1$ / C2-Facettenbeteiligung sowie die Beschreibung des Frakturverlaufs, der Dislokation und der Trümmerzone. Unter 52 Densfrakturen fand sich eine Übereinstimmung in wenigstens 5 von 7 Punkten in $70 \%$, der $\mathrm{K}$ Wert betrug 0,48 .

\section{Schraubenosteosynthese}

Operationstechnik. Sehr unterschiedliche Techniken wurden für die Stabilisierung der Densfrakturen beschrieben:

- dorsale Fusionsoperationen mit $\mathrm{Cl} /$ $\mathrm{C}$-Fusionen nach Brooks u. Jenkins [17] und Gallie [25] (• Abb. 2)

- $\mathrm{C} 1 / \mathrm{C}_{2}$-transartikuläre Verschraubungen [37]

- verschiedene Techniken mit dorsalen Klammern

Die direkte Versorgung mit Verschraubung hat eine deutlich höhere Fusionsrate gegenüber der konservativen Behandlung mit Halo und Orthesen [53] erreicht und bei den Typ-II-Frakturen gute Ergebnisse erzielt $[19,24,26,33,40]$. Das funktionelle Resultat ist durchweg gut [52]. Ihre fast zeitgleiche Erstbeschreibung geht auf Böhler [14, 15] und Nakanishi [41] zurück und basiert auf den Ideen von Magerl.
Vorbereitung. Die Lagerung erfolgt mittels Mayfield-Bügel, Halo-Ring und Crutchfield-Klemme und stellt den wichtigsten Teil des operativen Eingriffs dar. 2 Bildwandler sind für die Darstellung in jedem Fall zu fordern, um eine exakte präoperative Reposition und Darstellung im frontalen und sagittalen Strahlengang zu ermöglichen. Häufigste Ursache für technische Fehler sind eine falsch interpretierte, unvollständige Reposition und eine ungenaue röntgenologische Darstellung des Dens (• Abb. 3).

Die Vorbereitungsphase dauert häufig länger als die operative Phase, und es dürfen hierbei keinerlei Kompromisse gemacht werden, weil Korrekturen während der Operation oft nicht mehr möglich sind [12]. Bei der Einstellung der beiden Bildwandlergeräte kann es in der a.-p.Projektion u. U. dazu kommen, dass man einen Schneidezahn oder auch die Protuberantia occipitalis externa mit dem Dens verwechselt. Eine Verbesserung der Sicht auf den Dens kann durch Öffnung des Mundes während der Operation erreicht werden (• Abb. 4). In unserer Klinik wird dies mit einer aufgerollten elastischen Binde durchgeführt. Durch leichtes Kippen des Bildwandlers nach kranial kann die Darstellung verbessert werden.

Reposition der Fehlstellung. Sie erfolgt indirekt mit Hilfe der verschiedenen Einstellungen am Halo-Ring und Haltegerät. Eine Verschiebung kann durch die Bewegung des Kopfs ausgeglichen werden, die der Fehlstellung des Dens entgegenläuft: Extensionsbrüche werden also durch Flexion, Flexionsbrüche durch Extension der

- B1: Isthmusfrakture 


\section{Verletzungen der Halswirbelsäule}

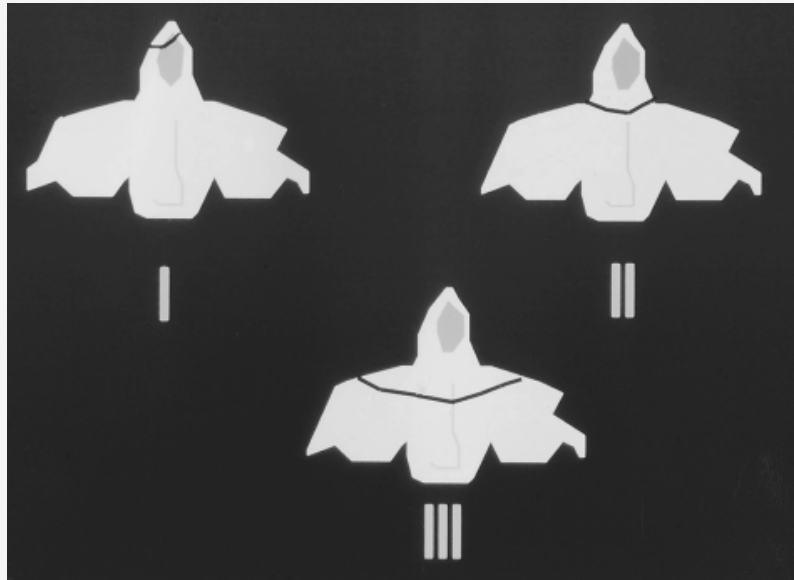

Abb. $1 \Delta$ Einteilung der Densfrakturen nach Anderson u. D’Alonzo [3]

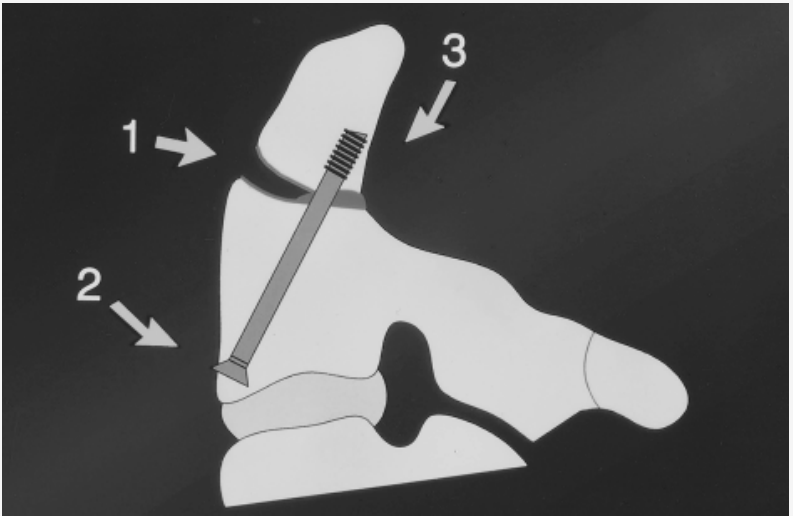

Abb. 3 \ Mögliche Probleme: bei Implantation der Schraube: 1. ungenügende Reposition, 2. zu ventrale Position der Schraube mit der Gefahr des Schraubenausbruchs bei erheblicher Osteoporose, 3. zu rasches Heraustreten der Schraube an der Denshinterfläche

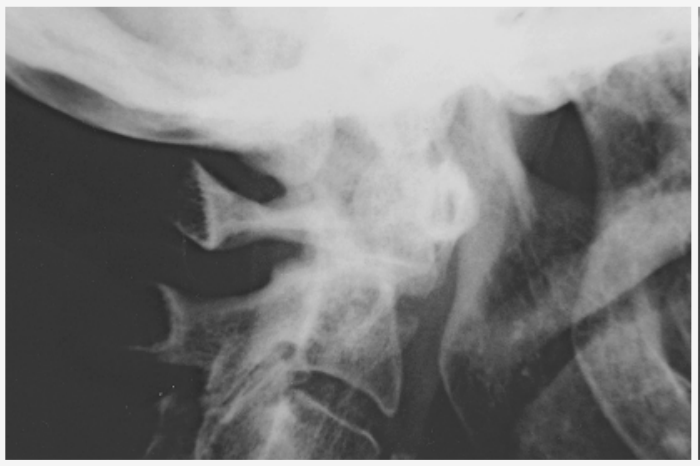

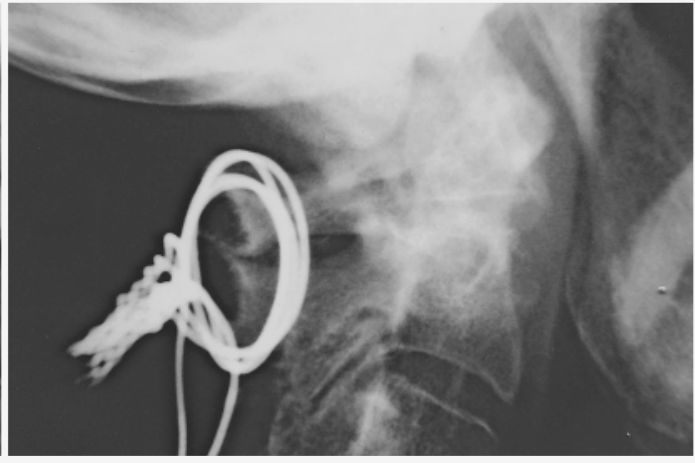

Abb. $2<$ Dorsale Spondylodese einer Pseudarthrose und Ausheilung

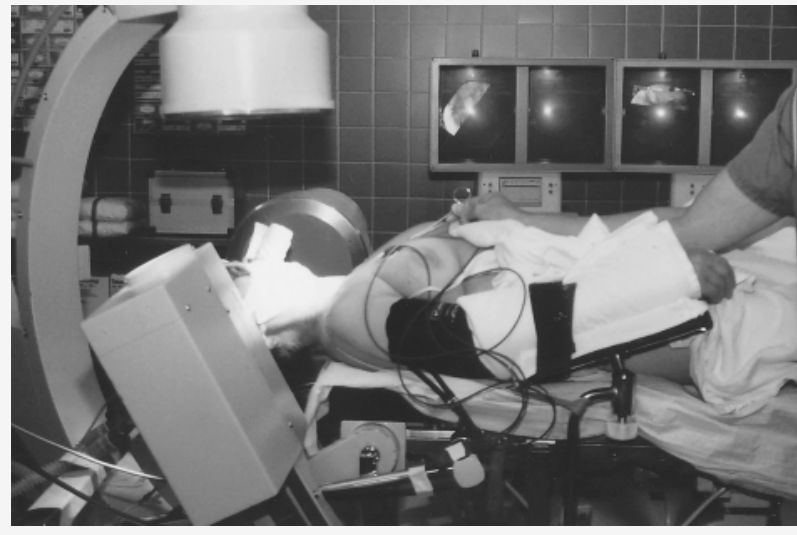

Abb. $4<$ Einstellung mit 2 Bildwandlern, Platzierung eines Kirschner-Drahts präoperativ zur Markierung der späteren Bohrrichtung, elastische Binde im Mund erlaubt bessere a.-p.-Darstellung
HWS reponiert [50]. Problematisch kann es bei Extensionsfrakturen werden, wenn die Flexion zur Folge hat, dass die Schrauben nicht mehr im notwendigen Winkel eingebracht werden können. Mit dem Haltegerät eröffnet sich jedoch die Möglichkeit, den Rumpf im Sinne einer sagittalen Translation nach vorne zu verschieben.

Nach Abschluss der Lagerung sollte auf die Fraktur eine leichte Kompression ausgeübt werden, um eine Schließung des Frakturspalts zu gewährleisten.
Zudem sollte unbedingt die so genannte Spickdrahtprobe durchgeführt werden, um zu sehen, ob das korrekte Platzieren der Schraube nicht dadurch verhindert wird, dass die Bohrmaschine nicht weit genug abgesenkt werden kann [50]. Dies ist nicht selten bei adipösen und emphysematösen Patienten der Fall. Der seitlich am Hals des Patienten angehaltene lange Spickdraht gibt einen Anhalt im seitlichen Strahlengang, ob die gewünschte Schraubenrichtung eingehalten werden kann.
Zwischen Spickdraht und vorderer Thoraxwand sollte mindestens ein Abstand von 1-2 cm liegen (• Abb. 4).

Operation. Der Zugang ist sowohl von rechts als auch von links möglich. Für den Rechtshänder hat der Zugang von rechts gewisse Vorteile. Er sollte ungefähr auf Höhe C6 liegen, um Bohrer und Schraube flach einbringen zu können. Liegt er höher, besteht die Gefahr, durch eine zu steile Bohrerführung die dorsale Kortika- 


\section{Zusammenfassung $\cdot$ Abstract}

lis des Dens schon kurz distal der Fraktur zu perforieren.

Für die einzelnen Operationsschritte sind inzwischen verschiedene Operationssets auf dem Markt [50]. Bei den kanülierten Schrauben sollte die Kortikalis der Densspitze durchbohrt sein, da hier, insbesondere auch beim alten Menschen, die Effizienz der Kompressionserhöhung am besten gewährleistet ist (• Abb. 5). Resorbierbare Schrauben zeigen in experimentellen Untersuchungen biomechanisch ein leichteres Bruchverhalten oder Verbiegung im Vergleich zu Titanschrauben [2].

Zahl der Schrauben. Während die Erstbeschreiber die Verwendung zweier Schrauben aufgrund der verbesserten Rotationsstabilität empfahlen, wiesen Doherty u. Heggenes [21], Nucci et al. [43] und $\mathrm{Xu}$ et al. [54] in anatomischen Studien nach, dass die Platzierung von 2 Schrauben in vielen Fällen nicht sicher möglich ist, da der Querdurchmesser des Dens axis die $8 \mathrm{~mm}$ für die Doppelgewindeschrauben und maximal 9 mm für kanülierte Schrauben häufig unterschreitet ( $\bullet$ Abb. 6$)$.

In biomechanischen Studien [28, 38] konnte keine verminderte Steifigkeit bei singulär eingebrachten Schrauben gegenüber der Doppelverschraubung nachgewiesen werden. Von vielen Autoren wird deshalb für die direkte Verschraubung nur noch eine Einzelschraube verwendet [18, 34,49 ].

\section{Komplikationen}

\section{Schraubenlockerung}

Das Durchschneiden der Schrauben aus dem Körper des HWK2 nach ventral ist häufig Folge einer zu ventralen Schraubenposition. Anderson et al. [5] berichteten bei 2 von 9 Patienten über dieses Problem. In den 2 Fällen war eine adäquate Schraubenposition bei korrekter Reposition nicht möglich, somit musste zur dorsalen $\mathrm{C}_{1} / \mathrm{C}_{2}$-Fusion gewechselt werden.

\section{Pseudarthrosen}

Obwohl der Begriff „Fusion“ kontrovers diskutiert wird, hat er innerhalb der Wirbelsäulenchirurgie eine enorme Bedeutung erlangt - insbesondere durch die

Trauma Berufskrankh $2005 \cdot 7$ [Suppl 2]: S271-S276

DOI 10.1007/s10039-005-1037-3

c) Springer Medizin Verlag 2005

\section{H.-J. Oestern}

\section{Komplikationen bei Densfrakturen}

\section{Zusammenfassung}

Bei Densfrakturen werden intra- und postoperative Komplikationen unterschieden. Erstere umfassen die unkorrekte Reposition, schlechte Übersichtlichkeit und Verletzungen von Halsorganen (A.-carotis-, Ösophagusverletzungen). Sie lassen sich durch exakte präoperative Reposition und Einstellung des Dens in beiden Ebenen mit Hilfe von 2 Bildwandlergeräten und die präoperative Markierung der Schraubenrichtung vermeiden. Postoperative Komplikationen umfassen die Pseudarthrosen und die progressive Myelopathie. Die Stabilisierung

Ersterer erfolgt mit Hilfe der transartikulären Verschraubung nach Magerl in Kombination mit einer Spondylodese nach Gallie oder Brooks. Die progressive Myelopathie hat ihre Ursache häufig in einer unzureichenden Reposition und damit einer Verringerung des Rückenmarkquerschnitts.

\section{Schlüsselwörter}

Unkorrekte Reposition .

Schlechte Übersichtlichkeit · Verletzungen von Halsorganen · Pseudarthrosen .

Progressive Myelopathie

\section{Complications following fracture of a tooth}

\section{Abstract}

A distinction is made between intra- and postoperative complications. Intraoperative complications embrace incorrect repositioning, a poor overall view and injuries to organs in the neck (the carotic artery or the oesophagus). These can be avoided by precise repositioning preoperatively and adjustment of the dens in both planes with the aid of two image converters, and preoperative marking of the orientation of the screws. Postoperative complications can take the form of pseudoarthrosis and/or progressive myelopathy. Pseudarthrosis is sta- bilised with the aid of transarticular screw fixation after Magerl in combination with fusion using the Gallie or the Brooks technique. Progressive myelopathy frequently has its roots in inadequate repositioning resulting in restriction of the diameter of the spinal cord.

\section{Keywords}

Incorrekte repositioning · Poor overall view . Injuries to organs in the necj.

Pseudarthroses · Progressive myelopathy 


\section{Verletzungen der Halswirbelsäule}
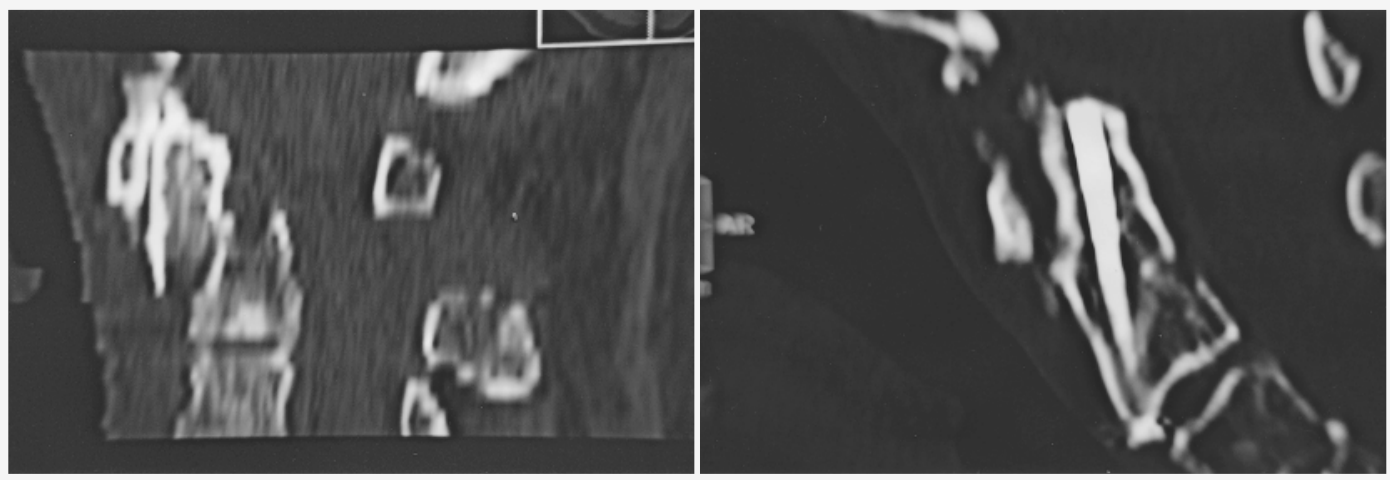

Abb. $5<$ CT-Rekonstruktion einer Anderson-II-Fraktur und Stabilisierung mit einer Schraube

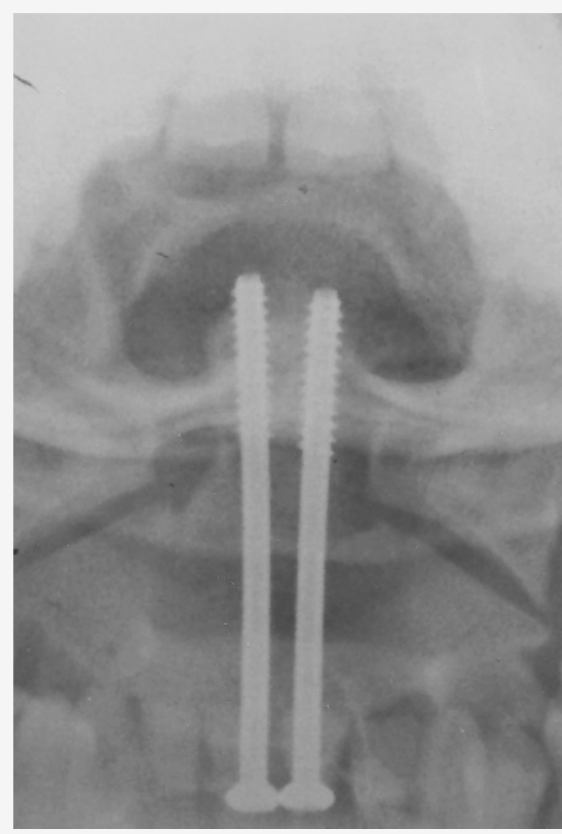

Schwierigkeiten in der Definition einer Fusion und deren Dokumentation (Fehlen verbindlicher radiologischer Kriterien, signifikante Interobserverunterschiede) [51]. Die gelungene Fusion repräsentiert das häufigste Kriterium zur Dokumentation des Erfolgs von Wirbelsäulenoperationen.

\section{Diagnostik von Denspseudarthrosen.}

Sie entspricht im Wesentlichen der der frischen Densfraktur. Zusätzlich zur konventionellen Röntgenaufnahme der HWS sollten Funktionsuntersuchungen in Flexion und Extension (Stabilität der Pseudarthrose) und eine computertomographische Untersuchung angeschlossen werden (Weite des Pseudarthrosenspalts, mögliche Fehlstellung der atlantoaxialen Gelenke) [12].

Einteilung. Die Denspseudarthrosen werden nach Stabilität, Dislokationsausmaß

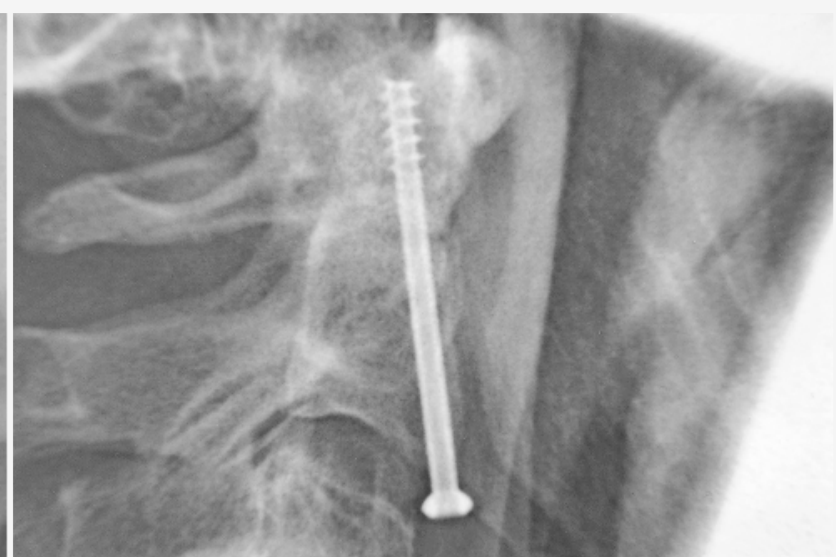

Abb. $6<$ Stabilisierung einer Densfraktur mit 1 bzw. 2 Schrauben, wichtig: Perforation der Schraube an der Densspitze

und Beschwerden in 4 verschiedene Typen klassifiziert. Die Fusionsraten in der neueren Literatur liegen bei $87 \%$ [24]. Aebi et al. [1] berichteten ebenso wie Apfelbaum et al. [6] über eine Fusionsrate von $88 \%$. Etter et al. [23] erzielten 32,3\% Fusionen, während El Saghir u. Böhm [22] über eine 100\%ige Fusionsrate berichteten. Auch die Zeitabläufe bis zur Fusion sind deutlich unterschiedlich und beliefen sich auf 11,2 Monate (zwischen 6 und 18 Monaten) in der Untersuchung von Fountas et al. [24], 8 Monate (zwischen 3 und 24 Monate) bei Aebi et al. [1] und 5,5 Monate bei Etter et al. [23].

In mehreren retrospektiven Untersuchungen konnte dargestellt werden, dass weder das Alter $[11,16]$ noch das Geschlecht die Heilungszeit entsprechend signifikant verändern bzw. beeinflussen. Apfelbaum et al. [6] bestätigten ebenfalls, dass diese beiden Parameter keinen statis- tisch signifikanten Einfluss auf das chirurgische Outcome haben.

Häufigkeit. Die Pseudarthroserate für Typ-II-Frakturen beläuft sich in der Literatur auf Werte zwischen 4,8\% und 62,8\% [4, $5,9,13,32,45,46,47,48,52]$. Bei verschobenen Frakturen erreicht sie sogar Werte zwischen $67 \%$ [31] und $88 \%$ [7].

Therapie. Ziel der operativen Behandlung der Denspseudarthrose sind die Reposition, Fusion und Stabilisierung. Als stabilste Methode gilt die dorsale $\mathrm{C}_{1} / \mathrm{C}_{2}$-Fusion nach Magerl u. Seemann [37], eine dorsale transartikuläre Verschraubung der atlantoaxialen Gelenke in Kombination mit einer Spondylodese im Sinne einer Fusion nach Gallie $[25,30]$.

Vorteile dieser Methode sind die hohe Stabilität hinsichtlich Rotation, Extension und Flexion. Die Gefahren sind einer- 
seits die Verletzung der A. vertebralis bei Präparation am lateralen Rand des atlantoaxialen Gelenks oder innerhalb der Foramina Processus transversi bei zu lateraler Bohrung [12], andererseits die Verletzung des parallel zu den Dornfortsätzen verlaufenden Venenplexus, des N. occipitalis major (R. dorsalis medialis des Spinalnervs T2) und von Rückenmark und Dura mater v. a. bei zu medialer Schraubenlage oder zu starker Schraubenkonvergenz.

Eine Alternative zu dieser Operationstechnik ist die okzipitozervikale Fusion mit Y-Platte nach Grob oder anderen Implantaten [29]. Nachteile dieser Methode sind Auftreten bzw. Verbleib von Pseudarthrosen und die Gefahr des Materialbruchs durch Mikrobewegungen und Hebelwirkungen des Hinterhaupts sowie der vorgegebene Abstand der Plattenlöcher. Weitere Komplikationen sind Liquoraustritt und venöse Blutungen bei Anbringen der Bohrlöcher am Schädel sowie die Fixation des Kopfs in starker Inklination mit nachfolgender Sichtbehinderung und Schluckstörungen durch Druck des Kinns auf den Kehlkopf oder durch Dauerirritation des Retropharynx bei zu lang gewählten transartikulären Schrauben [10]. Vorteile dieser Methode sind eine hohe multidirektionale Stabilität v. a. in Verbindung mit der transartikulären $\mathrm{C}_{1} / \mathrm{C}_{2}$-Verschraubung und eine Schraubenverankerung am Ort mit der durchschnittlich größten Knochendicke und somit größtmöglichem Halt.

Als großer Nachteil der dorsalen Fixation des okzipitalen Übergangs ist die hohe Einschränkung der Beweglichkeit der oberen Halswirbelsäule (HWS) zu nennen. Untersuchungen haben einen Verlust der Rotationsbeweglichkeit bis zu 6o\% gezeigt [44]. Durch kompensatorisches Drehen des Rumpfs kann diese Einschränkung gut ausgeglichen werden, bestimmte Tätigkeiten bleiben aber beeinträchtigt, z. B. Autofahren. Bei der atlantoaxialen Fusion ist diese Bewegungseinschränkung nicht so ausgeprägt. Es wurden Einschränkungen der Beweglichkeit um mindestens 25\% bei Rotation sowie bei Extension/Flexion in der oberen HWS und um mindestens $20^{\circ}$ bei Seitneigung des Kopfs gefunden [39]. Die okzipitozervikale Fusion ist meist bei Patienten mit reduziertem Allgemeinzustand indiziert.
Risikofaktoren für die Entstehung einer Pseudarthrose. Koivikko et al. [36] untersuchten an 69 Patienten, die mit dem Halo versorgt wurden, die Faktoren, die einen Einfluss auf die Pseudarthroserate hatten. Risikofaktoren für eine Pseudarthrose stellten ein Frakturspalt $>1 \mathrm{~mm}$, eine dorsale Verschiebung $>5 \mathrm{~mm}$, ein über 4 Tage verzögerter Behandlungsbeginn und eine wiederholte Verschiebung nach dorsal über $2 \mathrm{~mm}$ dar.

\section{Progressive Myelopathie}

Das späte Auftreten einer solchen ist nach wie vor eine Seltenheit. Crockard et al. [20] untersuchten 16 Patienten mit Densfrakturen 4 Monate bis 45 Jahre nach dem Unfall. 15 Patienten hatten charakteristische $\mathrm{C}_{2}$-Wurzelschmerzen und 10 ein Schwächegefühl in den Händen und Gehunsicherheiten bemerkt. 15 Patienten wiesen Typ-2-Frakturen auf, die in 11 Fällen mobil waren. Eine hypertrophe Pseudarthrose wurde bei 2 Patienten beobachtet, bei 7 Patienten wurde anlässlich der Operation bemerkt, dass ein Teil oder das ganze Lig. transversum in die Fraktur interponiert war. Über einen transoralen $\mathrm{Zu}$ gang in Kombination mit einer Vielzahl verschiedener dorsaler Fusionstechniken konnten eine Myelondekompression erreicht und die Symptomatik aufgehalten werden. Die Werte der kraniovertebralen Winkel und die Rückenmarkquerschnittsfläche zeigten eine Korrelation, aber das entscheidende Kriterium für das Auftreten der progressiven Myelopathie war das Verhältnis zwischen der Länge des Unfallzeitraums, der verzögerten Diagnose und dem verminderten Rückenmarkquerschnitt. Diese späte Myelopathie nach Densfrakturen scheint häufiger vorzukommen, als allgemein angenommen, insbesondere bei Patienten mit zervikaler Spondylose.

Bei einer Spinalkanaleinengung durch eine atlantoaxiale Pseudarthrose werden die transorale Resektion des Dens und atlantoaxiale Plattenstabilisierung empfohlen.

\section{Gefäßverletzungen}

Arand et al. [8] berichteten über einen Fall mit Abriss der A. carotis interna. Das Ge- fäß wickelte sich beim Bohren bei zu kurzem Gewindeschutz um das Instrument und zerriss. Bei dem 23-jährigen Patienten wurde ein prothetischer Ersatz mit gutem funktionellem Ergebnis vorgenommen.

\section{Ösophagusperforation}

Eine iatrogene Ösophagusperforation mit Ausbildung eines tiefen Weichteilinfekts wurde von Arand et al. [8] beschrieben. Der Einriss wurde verschlossen und der Infekt beherrscht.

Einen ähnlichen Verletzungsmechanismus beschrieben Nerot et al. [42] bei einem 55-jährigen Patienten mit Densfraktur (Typ II).

Kathrein et al. [35] berichteten über einen 30-jährigen Patienten mit einem Schädel-Hirn-Trauma nach einem Mountainbikeunfall. Die Primärbehandlung bestand in einer geschlossenen Reposition der Fraktur und Immobilisierung mit einem Stiff-neck. Am nächsten Tag erfolgte die operative Versorgung mittels zweier kanülierter Schrauben. Unmittelbar nach der Operation, nach Entfernung der Magensonde, wurde eine Blutung aus dem Mund beobachtet. Die Inspektion zeigte eine $4 \mathrm{~cm}$ lange Verletzung im Nasopharynx. Die daraufhin nochmals durchgeführte Betrachtung der präoperativen Röntgenbilder und des CT ergab neben einer retropharyngealen Schwellung anormale Lufteinschlüsse im prävertebralen Weichteilgewebe.

Die Betrachtung der Röntgenbilder auf Lufteinschlüsse, ebenso wie eine Blutung aus Mund und Nase sollten wegweisend sein im Hinblick auf eine Verletzung des Pharynx.

\section{Fazit}

Gerade bei der Densfraktur sind die präoperative Planung und Lagerung von ganz entscheidender Bedeutung. Nur bei präoperativer Reposition, der Verwendung von 2 Bildwandlern, der Anwendung der Kirschner-Draht-Methode zur Darstellung der Schraubenposition und der guten Sicht auf die Unterkante von C2 sind Komplikationen intraoperativer Art zu vermeiden. 


\section{Korrespondierender Autor Prof. Dr. H.-J. Oestern}

Klinik für Unfall- und

Wiederherstellungschirurgie,

Allgemeines Krankenhaus,

Siemensplatz 4, 29223 Celle

E-Mail: hans-joerg.oestern@akh-celle.de

Interessenkonflikt: Keine Angaben

\section{Literatur}

1. Aebi M, Etter C, Coscia M (1989) Fractures of the odontoid process - treatment with anterior screw fixation. Spine 14: 1065-1070

2. Ames $\mathrm{CP}$, Crawford NR, Chamberlain RH et al. (2005) Biomechanical evaluation of a bioresorbable odontoid screw. J Neurosurg Spine 2: 182-187

3. Anderson LD, D'Alonzo RT (1974) Fractures of the odontoid process of the axis. J Bone Joint Surg Am 56: 1663

4. Anderson LD, Clark CR (1989) Fractures of the odontoid process of the axis. In: Sherk HH (ed) The cervical spine. Lippincott, Williams \& Wilkins, Philadelphia, PA

5. Anderson S, Rodrigues M, Olerud C (2000) Odontoid fractures: high complication rate associated with anterior screw fixation in the elderly. Eur Spine J 9: 56-60

6. Apfelbaum RI, Lonser RR, Veres R et al. (2000) Direct anterior screw fixation for recent and remote odontoid fractures. J Neurosurg Spine 93: 227-236

7. Apuzzo MLJ, Heiden JS, Weiss HM et al. (1978) Acute fractures of the odontoid process: an analysis of 45 cases. J Neurosurg 48: 85-91

8. Arand M, Lemke M, Kinzl L et al. (2001) Omzidenz von Komplikationen der Schraubenosteosynthese von Frakturen des Dens axis. Zentralbl Chir 126 610-615

9. Aymes EW, Anderson FM ( 1956) Fracture of the odontoid process. Arch Surg 72: 377-393

10. Bading S, Pape HC, Lange U et al. (2004) Reosteosynthesis in dens pseudarthrosis. Case report and review of the literature. Unfallchirurg 107: 149153

11. Berlemann U, Schwarzenbach O (1997) Dens fractures in the elderly. Acta Orthop Scand 68: 319324

12. Blauth M, Richter M, Kiesewetter B et al. (1999) Operative oder konservative Behandlung der Pseudarthrose des Dens axis: Wie gefährlich ist es, eine Denspseudarthrose nicht zu stabilisieren? Chirurg 70: 1225-1238

13. Blockley NJ, Purser DW (1956) Fractures of the odontoid process of the axis. J Bone Joint Surg $\mathrm{Br}$ 38: 794

14. Böhler J (1981) Schraubenosteosynthese von Frakturen des Dens axis 1981. Unfallheilkunde 84: 221223

15. Böhler J (1982) Anterior stabilization for acute fractures and non-unions of the dens. J Bone Joint Surg Am 64: 18-27

16. Börm W, Kast E, Richter HP et al. (2003) Anterior screw fixation in type II odontoid fractures: is there a difference in the outcome between age groups? Neurosurgery 53: 1089-1094

17. Brooks Al, Jenkins EB (1978) Atlanto-axial arthrodesis by he wedge compression method. J Bone Joint Surg Am 60: 279-284
18. Chang KW, Liu YW, Cheng PG et al. (1994) One Herbert double-threaded compression screw fixation of displaced type Il odontoid fractures. J Spinal Disord 7: 62-69

19. Chiba K, Fujimura $Y$, Toyama $Y$ et al. (1993) Anterior screw fixation for odontoid fracture: clinical results in 45 cases. Eur Spine J 2: 76-81

20. Crockard HA, Heilman AE, Stevens JM (1993) Progressive myelopathy secondary to odontoid fractures: clinical, radiological, and surgical features. J Neurosurg 78: 579-586

21. Doherty BJ, Heggenes MH (1995) Quantitative anatomy of the second cervical vertebra. Spine 20 : 513-317

22. ElSaghir H, Böhm H (2000) Anderson type II fracture of the odontoid process: results of anterior screw fixation. J Spinal Disord 13: 527-530

23. Etter C, Coscia M, Jaberg H et al. (1991) Direct anterior fixation of dens fractures with a cannulated screw system. Spine 16: 25-32

24. Fountas K, Kapsalaki E, Karampelas I et al. (2005) Results of long-term follow-up in patients undergoing anterior screw fixation for type II and rostral type III odontoid fractures. Spine 15: 661-669

25. Gallie WE (1939) Fractures and dislocations of the cervical spine. Am J Surg 46: 495-499

26. Geisler R, Cheng C, Poka A et al. (1989) Anterior screw fixation of posteriorly displaced type II odontoid fractures. Neurosurgery 25: 30-38

27. Grauer JN, Shafi B, Hilibrand AS et al. (2005) Proposal of a modified, treatment-oriented classification of odontoid fractures. Spine J 5: 123-129

28. Graziano G, Jaggers C, Lee M et al. (1993) A comparative study of fixation techniques for type II fractures of the odontoid process. Spine 18: 2383-2387

29. Grob D (1992) Dorsale Fixation des okzipitozervikalen Übergangs. Operat Orthop Traumatol 4: 151160

30. Grob D, Jeanneret B, Aebi M et al. (1991) Atlanto-axial fusion with transarticular srew fixation. J Bone Joint Surg Br 73: 972

31. Hadley MN, Browner C, Sonntag VK (1985) A comprehensive review of management and treatment of 107 cases. Neurosurgery 17: 281-290

32. Hadley MN, Browner CM, Liu SS et al. (1988) New subtype of acute odontoid fractures (type IIA). Neurosurgery 22: 67-71

33. Henry AD, Bohly J, Grosse A (1999) Fixation of odontoid fractures by an anterior screw. J Bone Joint Surg Br 81: 472-477

34. Jenkins JD, Coric D, Branch CL Jr (1998) A clinical comparison of one- and two-screw odontoid fixation. J Neurosurg 89: 366-370

35. Kathrein A, Genelin A, Gupta R et al. (2000) Odontoid fracture associated with a pharyngeal tear. A case report. J Bone Joint Surg Am 82: 1154-1156

36. Koivikko MP, Kiuru MJ, Koskinen SK et al. (2004) Factors associated with nonunion in conservatively-treated type-II fractures of the odontoid process. J Bone Joint Surg Br 86: 1146-1151

37. Magerl, Seemann PS (1986) Stable posterior fusion of the atlas and axis by transarticular srew fixation. In: Kehr P, Weidner A (eds) Cervical spine. Springer, Berlin Heidelberg New York, pp 322-327

38. McBride AD, Mukherjee DP, Kruse RN et al. (1995) Anterior screw fixation of type II odontoid fractures. A biomechanical study. Spine 20: 1855-1860

39. Mestdagh H, Vigier P, Berger M et al. (1988) Résultats de l'arthrodèse atloido-axoidienne avec cerclage postérieur pour fracture de l'odontoide. A propos de cinquante-neuf cas. Ann Chir 42: 492-500

40. Montesano P, Anderson P, Schlehr F et al. (1991) Odontoid fractures treated by anterior odontoid screw fixation. Spine 16: 33-37
41. Nakanishi T (1980) Internal fixation of odontoid fracture (in Japanese). Orthop Trauma Surg 23: 399-406

42. Nerot C, Jeanneret B, Lardenois T et al. (2002) Esophageal perforation after fracture of the cervical spine: case report and review of the literature. J Spinal Disord Tech 15: 513-518

43. Nucci RC, Seigal S, Merola AA et al. (1995) Computed tomographic evaluation of the normal adult odontoid. Implications for internal fixation. Spine 20: $264-270$

44. Penning L, Wilmink JT (1987) Rotation of the cervical spine: A CT study in normals. Spine 12: $726-$ 731

45. Roberts A, Wickstrom J (1972) Prognosis of odontoid fractures. Proceedings of The American Academy of Orthopedic Surgeons. J Bone Joint Surg Am 54: 1353

46. Schatzker J, Rorabeck CH, Waddell JP (1971) Fractures of the dens (odontoid process): an analysis of thirty-seven cases. J Bone Joint Surg 53: 392-405

47. Schatzker J, Rorabeck CH, Waddell JP (1975) Nonunion of the odontoid process. An experimental investigation. Clin Orthop 108: 127-137

48. Southwick WO (1980) Management of fractures of the dens (odontoid process). J Bone Joint Surg Am 62: $482-486$

49. Subach BR, Morone MA, Haid RW Jr et al. (1999) Management of acute odontoid fractures with single-screw anterior fixation. Neurosurgery 45: $812-$ 820

50. Tscherne H, Blauth M (1998) Unfallchirurgie Wirbelsäule. Springer, Berlin Heidelberg New York

51. Tuli SK, Chen P, Eichler ME et al. (2004) Reliability of radiologic assessment of fusion: cervical fibular allograft model. Spine 14: 856-860

52. Verheggen R, Jansen J (1994) Fractures of the odontoid process: analysis of the functional results after surgery. Eur Spine J 3: 146-150

53. Wang G-J, Mabie KN, Whitehill R et al. (1984) The nonsurgical management of odontoid fractures in adults. Spine 9: 229-230

54. Xu R, Nadaud MC, Ebraheim NA et al. (1995) Morphology of the second cervical vertebra and the posterior projection of the $\mathrm{C} 2$ pedicle axis. Spine 20: $259-263$ 